\title{
Negative Domain Wall Contribution to the Resistivity of Microfabricated Fe Wires
}

\author{
U. Ruediger, J. Yu, S. Zhang, and A. D. Kent* \\ Department of Physics, New York University, 4 Washington Place, New York, New York 10003 \\ S. S. P. Parkin \\ IBM Research Division, Almaden Research Center, San Jose, California 95120-6099
}

\begin{abstract}
The effect of domain walls on electron transport has been investigated in microfabricated Fe wires ( 0.65 to $20 \mu \mathrm{m}$ linewidths) with controlled stripe domains. Magnetoresistance (MR) measurements as a function of domain wall density, temperature, and the angle of the applied field are used to determine the low field MR contributions due to conventional sources in ferromagnetic materials and that due to the erasure of domain walls. A negative domain wall contribution to the resistivity is found. This result is discussed in light of a recent theoretical study of the effect of domain walls on quantum transport.
\end{abstract}

PACS numbers: 73.61.-r, 73.20.Fz, 75.60.Ch

The interplay between the electron transport and magnetic properties of ferromagnetic nanowires and thin films has recently been the subject of an intense research effort. In mesoscopic ferromagnets an experimental aim has been to use magnetoresistance (MR) to study domain wall (DW) dynamics, in particular, macroscopic quantum tunneling. These experiments have focused on the low temperature MR of nanowires of Ni [1], Co, and Fe [2]. Discontinuous changes in the wire conductance are observed as a function of the applied field. These are interpreted as the nucleation and movement of DWs which traverse the wire during magnetization reversal. In these experiments, nucleation of a DW appears to lead to a decrease in the wire's resistivity. Independently a novel theoretical explanation has been proposed in which DWs destroy the electron coherence necessary for weak localization at low temperature, leading to such a negative DW contribution to the resistivity [3]. Another recent experiment suggests large MR effects due to DWs can be observed even at room temperature in simple ferromagnetic films [4]. A new physical mechanism has been proposed to explain these observations which is analogous to that operative in giant magnetoresistance (GMR). Within this model the resistivity in the presence of DWs is enhanced due to a mixing of minority and majority spin channels in a wall in the presence of spin dependent electron scattering $[5,6]$. This research points to the need for experiments over a range of temperatures on ferromagnetic wires with well characterized and controllable domain patterns to isolate the important contributions to the MR in small samples.

Here we report on such experiments. Epitaxially oriented micron scale $\mathrm{Fe}$ wires with controlled domain configurations have been realized to study the effect of DWs on magnetotransport properties. In order to isolate the DW contribution to the MR the conventional sources of low temperature MR in ferromagnets are characterized in detail - both the anisotropic magnetoresistance (AMR) and Lorentz MR. As preliminary experiments on ferromag- netic nanowires suggest $[1,2]$, we find that DWs enhance the wire conductance at low temperature. This remarkable effect, present in micron scale wires, is difficult to reconcile with the existing theories of DW scattering.

The starting point for these experiments are high quality thin (100 nm thick) epitaxially grown (110) oriented bcc Fe films. These films display a large in-plane uniaxial magnetocrystalline anisotropy, with the easy axis parallel to the [001] direction. They are grown on sapphire substrates as described in Ref. [7]. The films are patterned using projection optical lithography and ion milling to produce micron scale wires $(0.65$ to $20 \mu \mathrm{m}$ linewidths of $\sim 200 \mu \mathrm{m}$ length) and wire arrays $(0.65$ to $20 \mu \mathrm{m}$ linewidths of $3 \mathrm{~mm}$ length and 10 to $20 \mu \mathrm{m}$ spacing) with the wires oriented perpendicular to the magnetic easy axis and parallel to the [1]0] direction. The residual resistivity ratio of 30 and the residual resistance $\rho_{0}=0.2 \mu \Omega \mathrm{cm}$ attest to the high crystalline quality of these films.

The competition between magnetocrystalline, exchange, and magnetostatic interactions results in a pattern of regularly spaced stripe domains perpendicular to the wire axis. Varying the wire linewidth changes the ratio of these energies and hence the domain size. Figure 1 shows magnetic force microscopy (MFM) measurements of a $2 \mu \mathrm{m}$ wire in zero field performed at room temperature with a vertically magnetized tip. These images highlight the DWs and magnetic poles at the wire edges. For instance, clearly visible in Fig. 1(b) are light and dark contrast along the DWs indicative of Bloch-like walls with sections of different chirality. The magnetic domain configurations are strongly affected by the magnetic history of the samples. Before imaging the wires were magnetized to saturation with a magnetic field transverse [Fig. 1(a)] or longitudinal [Fig. 1(b)] to the wire axis. In the transverse case the mean stripe domain length is $1.6 \mu \mathrm{m}$ and much larger than in the longitudinal case, where it is $0.4 \mu \mathrm{m}$. The observed domain structure at $H=0$ is stable over observation times 

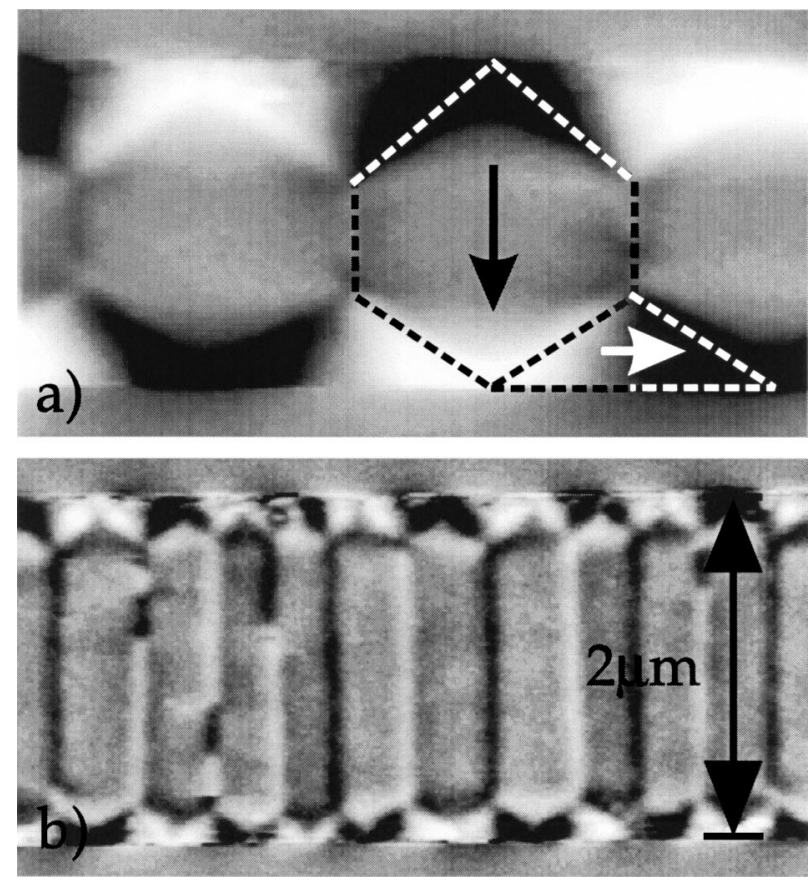

FIG. 1. MFM images in zero applied field of a $2 \mu \mathrm{m}$ linewidth Fe wire. Before performing the MFM images the wire was magnetized (a) transverse and (b) longitudinal to the wire axis.

of at least several hours showing that the DWs are strongly pinned at room temperature.

In Fig. 2 the average domain wall separation is plotted as a function of wire linewidth and magnetic history. The DW density varies by an order of magnitude for the linewidths investigated. Differences between domain configurations after transverse and longitudinal saturation are observed for wires with linewidths between 1 and $10 \mu \mathrm{m}$. Dotted lines in Fig. 1(a) illustrate the approximate domain structure. Since current is directed along the wire, there are domains with magnetization $\mathbf{M}$ oriented both parallel and perpendicular to the current density $\mathbf{J}$. In order to estimate the MR contributions due to resistivity anisotropy the volume fraction of closure domains (with $\mathbf{M} \| \mathbf{J}$ ) has been estimated. Figure 2 also shows this fraction (labeled $\gamma$ ) determined from MFM images after magnetic saturation in either the transverse or longitudinal direction.

MR measurements were performed in a variable temperature high field cryostat with in situ (low temperature) sample rotation capabilities. The applied field was in the plane of the film and oriented either longitudinal (II) or transverse $(\perp)$ to the wire axis. A 4 probe ac $(\sim 10 \mathrm{~Hz})$ resistance bridge with low bias currents (10 to $40 \mu \mathrm{A}$ ) was employed, and the magnetic history of the sample was carefully controlled. Figure 3 shows representative MR results on a $2 \mu \mathrm{m}$ linewidth wire at both (a) high $(270 \mathrm{~K})$ and (b) low temperature $(1.5 \mathrm{~K})$. There is structure to the MR in applied fields less than the satura-

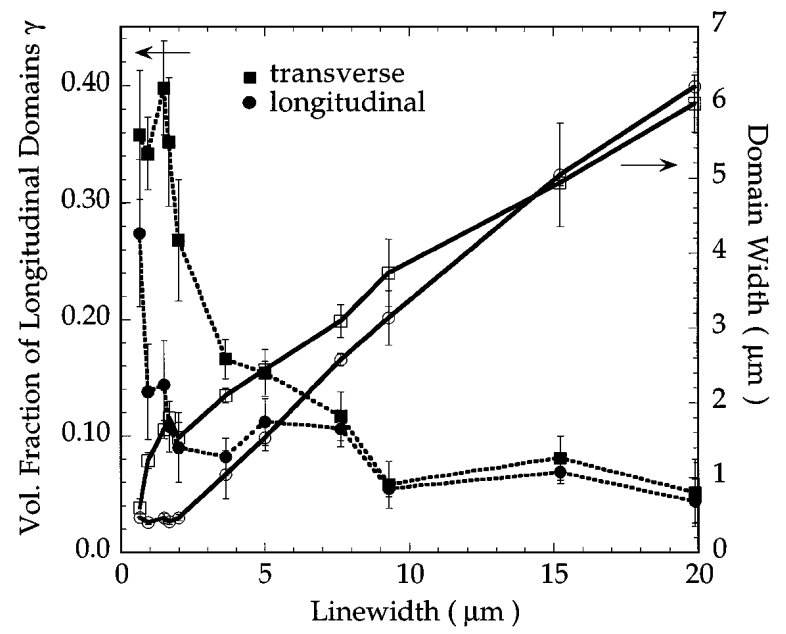

FIG. 2. The right-hand axis displays the domain width versus Fe linewidth in zero field after transverse (open squares) and longitudinal (open circles) magnetic saturation. The left-hand axis shows the volume fraction of closure domains $\gamma$ as function of the linewidth, again, after transverse (solid squares) and longitudinal (solid circles) magnetization.

tion field $\left(H_{s \|}=0.035 \mathrm{~T}\right.$ and $\left.H_{s \perp}=0.085 \mathrm{~T}\right)$, at which point the MR slope changes, and the resistivity then increases monotonically with field. At $270 \mathrm{~K}$ the resistivity above the saturation field is larger in the longitudinal than in the transverse field orientation, while at $1.5 \mathrm{~K}$ this resistivity anisotropy is reversed, $\rho_{\perp}\left(H_{s}\right)>\rho_{\|}\left(H_{s}\right)$.

Evidently there are competing sources of resistivity anisotropy in these films which leads to this reversal of the resistivity anisotropy with temperature. Two predominant

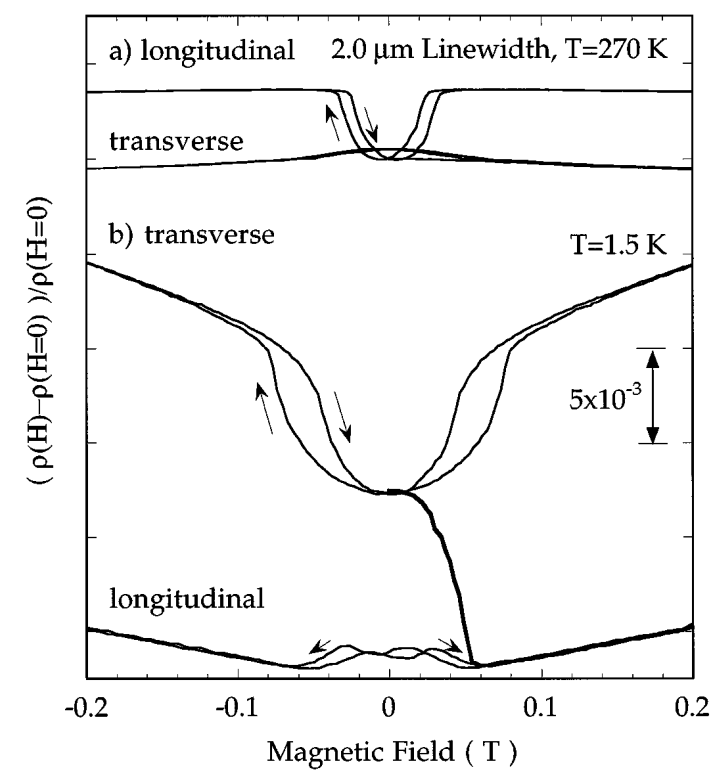

FIG. 3. (a) MR data at $270 \mathrm{~K}$ of a $2 \mu \mathrm{m}$ wire in the transverse and a longitudinal field geometries $\left[\rho_{\perp}(H=0,270 \mathrm{~K})=\right.$ $14.7 \mu \Omega \mathrm{cm}]$. (b) $\mathrm{MR}$ at $1.5 \mathrm{~K}$ again in the longitudinal and transverse field geometries $\left[\rho_{\|}(H=0,1.5 \mathrm{~K})=0.74 \mu \Omega \mathrm{cm}\right]$. 
and well understood sources of low field low temperature MR must be considered to interpret this transport data. The first has its origins in spin-orbit coupling and is known as AMR - the resistivity extrapolated back to zero internal field $(B=0)$ depends on the relative orientation of $\mathbf{M}$ and $\mathbf{J}$ [8]. The second effect is due to the ordinary (Lorentz) magnetoresistance and is also in general anisotropic (i.e., dependent on relative orientation of $\mathbf{J}$ and $\mathbf{B}$ ) [9]. As Fe has a large magnetization and hence a large internal magnetic field $(4 \pi M=2.2 \mathrm{~T})$ both factors are of importance. The resistivity of domains parallel and perpendicular to the current direction can be written as

$$
\begin{gathered}
\rho_{\perp}(B, T)=\rho_{\perp}(0, T)\left[1+F_{\perp}\left(B / \rho_{\perp}(0, T)\right)\right], \\
\rho_{\|}(B, T)=\rho_{\|}(0, T)\left[1+F_{\|}\left(B / \rho_{\|}(0, T)\right)\right] .
\end{gathered}
$$

Here $B$ is the internal field in the ferromagnet; $B=$ $4 \pi M+H-H_{d}$, with $H$ the applied field and $H_{d}$ the demagnetization field. The AMR is equal to $\left[\rho_{\|}(0, T)-\right.$ $\left.\rho_{\perp}(0, T)\right] / \rho(0, T)$, where $\rho(0, T)$ is the average resistivity. The function $F$ is known as the Kohler function and parametrizes the ordinary magnetoresistance for longitudinal and transverse field geometries in terms of $B / \rho \sim \omega_{c} \tau$, the cyclotron frequency times the relaxation time [10]. These scaling functions have been determined experimentally by performing MR measurements to large fields $(6 \mathrm{~T})$ as a function of temperature, as described in Ref. [9]. The scaling relationships [Eqs. (1) and (2)] are shown in Fig. 4. The inset displays both $\rho_{\perp}(0, T)$ and $\rho_{\|}(0, T)$ which result from this scaling analysis and which overlap on the scale shown. We find $\rho(0, T) \sim a T^{2}$ with $a=3 \times 10^{-4} \mu \Omega \mathrm{cm} / \mathrm{K}^{2}$, as typically observed in $3 d$

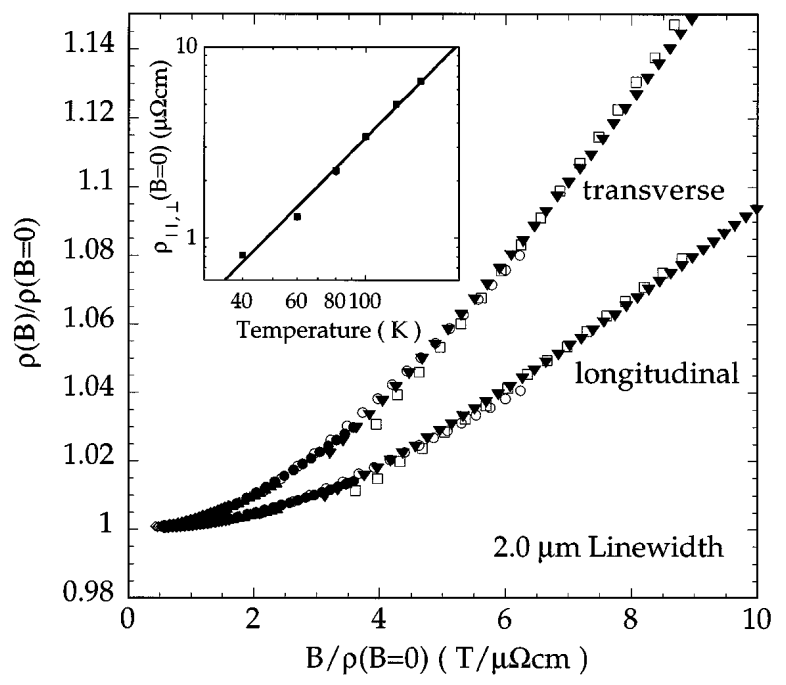

FIG. 4. Scaling plot of the transverse and longitudinal MR above magnetic saturation for a $2 \mu \mathrm{m}$ Fe wire in the form $\rho(B) / \rho(B=0)$ versus $B / \rho(B=0)$ at temperatures of (open squares) $1.5 \mathrm{~K}$, (solid triangles down) $40 \mathrm{~K}$, (open circles) $60 \mathrm{~K}$, (solid circles) $80 \mathrm{~K}$, (solid triangles up) $100 \mathrm{~K}$, (solid diamonds) $125 \mathrm{~K}$, and (open diamonds) $150 \mathrm{~K}$. The inset shows the scaling parameters $\rho_{\|}(B=0)$ and $\rho_{\perp}(B=0)$ as a function of temperature on a log-log plot. elemental ferromagnets [8]. The AMR is $\sim 4 \times 10^{-3}$ above $80 \mathrm{~K}$ and decreases below this temperature. The reversal of the resistivity anisotropy at low temperatures $\left[\rho_{\perp}\left(H_{s}\right)>\rho_{\|}\left(H_{s}\right)\right.$, Fig. 3(b)] is thus mainly a consequence of the increasing importance of the Lorentz MR (i.e., $\left.F_{\perp}^{\prime}>F_{\|}^{\prime}\right)$. At high temperature $\rho(0, T)$ is large and $F^{\prime}(x)_{x \rightarrow 0} \rightarrow 0$, so that the resistivity anisotropy is associated with the AMR as seen in Fig. 3(a).

As in all ferromagnetic materials the resistivity anisotropy is a source of low field MR. An applied field changes the domain configurations, and domains with magnetization parallel and perpendicular to the current direction have different resistivities. Hence, this low field MR simply reflects the domain geometries during magnetization.

There are thus two ways to estimate the DW contribution to the resistivity. The first is to perform MR measurements at the temperature at which this resistivity anisotropy at $H=0$ vanishes. Since the AMR and Lorentz MR contributions to the resistivity anisotropy are of opposite sign, there will be a temperature at which $\rho_{\|}\left(H=0, T_{\text {comp }}\right)=\rho_{\perp}\left(H=0, T_{\text {comp }}\right)$, which we denote the compensation temperature, $T_{\text {comp. }}$. This occurs at $65.5 \mathrm{~K}$ and MR results are shown in Fig. 5 for a $2 \mu \mathrm{m}$ wire. At this temperature the low field MR due to the resistivity anisotropy approaches zero. However, the measured resistivity at $H=0$ is lower in longitudinal than in the transverse field orientation. This correlates with DW density, which is larger after longitudinal magnetic

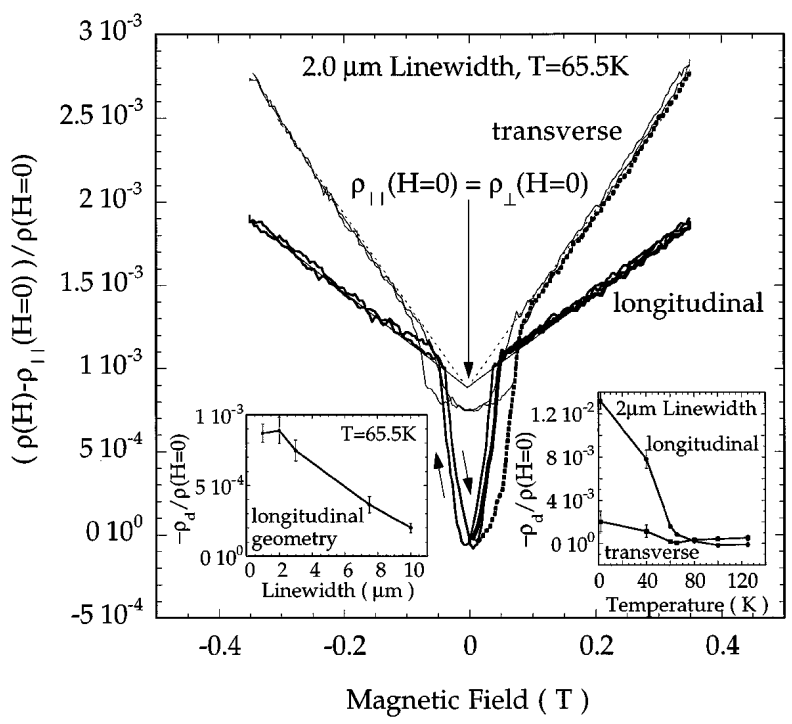

FIG. 5. MR of a $2 \mu \mathrm{m} \mathrm{Fe}$ wire at $65.5 \mathrm{~K}$. The extrapolation of the high field MR data in transverse (dotted line) and longitudinal (solid line) geometry shows that $\rho_{\perp}(H=0)=$ $\rho_{\|}(H=0)$. The resistivity with walls present, $\rho(H=0)$, is smaller than this extrapolation and indicates that DWs lower the wire resistivity. The left-hand inset shows this negative DW contribution as a function of linewidth at this compensation temperature in the longitudinal geometry. The right-hand inset shows the DW contribution as a function of temperature deduced using the model described in the text. 
saturation [Fig. 1(b)]. The magnitude of the effect also decreases systematically with increasing wire linewidth, (Fig. 5, left-hand inset) and, hence, decreasing DW density (Fig. 2). The observed resistivity at $H=0$ is apparently suppressed in the presence of DWs with a magnitude which depends on the density of DWs.

A more definitive correlation between domain configurations, measured at room temperature using an MFM, and low temperature MR measurements has been established. To do this we warm the sample to room temperature, cycle the magnetic field to establish a known $H=0$ magnetic state, and cool. The resistivity at $H=0$ and the MR at low temperatures are unchanged for these samples in both longitudinal and transverse measurement geometries. This is strong evidence that the domain structure is not affected by temperature in this range and consistent with temperature dependent magnetic hysteresis-loop measurements on wire arrays which show no change of the remanent magnetization with temperature.

The temperature dependence of the DW contribution to the resistivity is estimated as follows. The effective resistivity in the $H=0$ magnetic state due to resistivity anisotropy can be written as [11]

$$
\rho_{\mathrm{eff}}(H=0, T)=\gamma \rho_{\|}\left(B_{i}, T\right)+(1-\gamma) \rho_{\perp}\left(B_{i}, T\right),
$$

where $\gamma$ is the volume fraction of domains oriented longitudinally (see Fig. 2) and $B_{i}$ is the field internal to these domains $\left(=4 \pi M-H_{d}\right)$. We determine $\rho_{\perp}\left(B_{i}, T\right)$ and $\rho_{\|}\left(B_{i}, T\right)$ by extrapolation of the MR data above saturation (again, as indicated by the dashed and solid lines in Fig. 5). The effective resistivity at $H=0$ is estimated with the MFM measurements of $\gamma$. Deviations from this $\rho_{d}=\rho(H=0)-\rho_{\text {eff }}(H=0)$, i.e., the measured $H=0$ resistivity minus this effective resistivity, are negative and depend systematically on domain wall density, increasing in magnitude with increasing domain wall density. They approach $1.3 \%$ of the wire resistivity at $1.5 \mathrm{~K}$ for a $2 \mu \mathrm{m}$ linewidth wire. We also find that $\left|\rho_{d}\right|$ decreases with increasing temperature approaching zero at $\sim 80 \mathrm{~K}$ (Fig. 5, right-hand inset). This enhancement of the conductivity vanishes at $\sim 80 \mathrm{~K}$ for all the wire linewidths investigated.

There are a few models of DWs scattering which predict enhancements in the conductivity in the presence walls. One is that of Tatara and Fukuyama based in weak localization phenomena [3]. They find that DWs contribute to the decoherence of conduction electrons which destroys weak localization. They introduce a wall decoherence time to parametrize this effect $\tau_{w}=\tau /\left[n_{w} /\left(6 \lambda k_{f}^{2}\right)\left(\epsilon_{f} / \Delta\right)^{2}\right]$. Here $\tau$ is the momentum relaxation time, $n_{w}$ the domain wall density, $k_{f}$ the Fermi vector, $\lambda$ the domain wall thickness, and $\epsilon_{f} / \Delta$ the ratio of the Fermi energy to the exchange splitting of the band. With commonly used parameters for $s$ electrons in Fe, $\epsilon_{f} / \Delta \sim 500$, $k_{f} \sim 1.7 \AA^{-1}, \lambda \sim 300 \AA$, and with $n_{w}=2.5 \mu \mathrm{m}^{-1}$ we estimate $\tau_{w} \sim 60 \tau$. Essential to observing such an effect is the absence of other decoherence mechanisms, such as inelastic scattering. Equating $\tau_{w}=\tau_{\text {in }}$ gives an upper temperature limit for the presence of weak localization phenomena. From the residual resistance $\tau=2.8 \times 10^{-14} \mathrm{~s}$ and with $\rho_{\text {in }}=\alpha T^{2}$ $\left(\alpha=3 \times 10^{-4} \mu \Omega \mathrm{cm} / \mathrm{K}^{2}\right)$ we find $T_{\max }=7 \mathrm{~K}$. From this point of view the suppression of weak localization due to DWs cannot explain our observation of enhanced conductivity up to $\sim 80 \mathrm{~K}$.

In summary, a new lithographic approach has been used to realize ferromagnetic wires with controlled magnetic interactions and hence domain configurations. This has enabled a detailed investigation of the low field MR in micron scale ferromagnetic wires and, in particular, a study of the effect of DWs on the resistivity. After considering the effects of conventional sources of low field MR (AMR and the Lorentz MR), a negative DW contribution to the resistivity is identified. While a negative contribution is consistent with a recent theory based on weak localization, results above $\sim 10 \mathrm{~K}$ are difficult to reconcile with this theory. Further research of this type, on well characterized samples, is clearly warranted to elucidate the interplay between the transport and magnetic properties of mesoscopic ferromagnets.

The authors thank Peter M. Levy for helpful discussions of the work and comments on this manuscript. This research was supported by DARPA-ONR, Grant No. N00014-96-1-1207. We thank C. Noyan for x-ray characterization and $\mathrm{M}$. Ofitserov for technical assistance. Microstructures were prepared at the CNF, Project No. 588-96.

*Electronic address: andy.kent@ nyu.edu

[1] K. Hong and N. Giordano, Phys. Rev. B 51, 9855 (1995).

[2] Y. Otani et al., in Proceedings of the MRS Spring Meeting, San Francisco, 1997 (MRS, Warrendale, 1997).

[3] G. Tatara and H. Fukuyama, Phys. Rev. Lett. 78, 3773 (1997).

[4] J. F. Gregg et al., Phys. Rev. Lett. 77, 1580 (1996).

[5] M. Viret et al., Phys. Rev. B 53, 8464 (1996).

[6] P. M. Levy and S. Zhang, Phys. Rev. Lett. 79, 5110 (1997).

[7] A. D. Kent, U. Ruediger, J. Yu, S. Zhang, P. M. Levy, and S. S. P. Parkin, e-print cond-mat/9803101.

[8] See, e.g., I. A. Campbell and A. Fert, in Ferromagnetic Materials, edited by E. P. Wohlfarth (North-Holland Pub. Co., Amsterdam, 1982), Vol. 3.

[9] F. C. Schwerer and J. Silcox, Phys. Rev. Lett. 20, 101 (1968); J. Appl. Phys. 39, 2047 (1968).

[10] See, e.g., J. M. Ziman, Electrons and Phonons (Clarendon Press, Oxford, England, 1960), p. 490.

[11] Since the resistivity anisotropy is small, the current density in each domain is to a good approximation independent of the precise domain configurations. We also assume that the domain size is much greater than the mean free path. 